\title{
Expression levels of cytokines and chemokines increase in human peripheral blood mononuclear cells stimulated by activation of the Toll-like receptor 5 pathway
}

\author{
YU MA ${ }^{1}$, LI ZHANG ${ }^{2}$ and QUANSHENG $\mathrm{LI}^{3}$ \\ ${ }^{1}$ Department of Thyroid and Breast Surgery; ${ }^{2}$ Laboratory of Pathology, Department of Pathology; \\ ${ }^{3}$ Department of Biliary Surgery, West China Hospital, Sichuan University, Chengdu, Sichuan 610041, P.R. China
}

Received May 30, 2014; Accepted January 26, 2015

DOI: $10.3892 /$ etm.2015.2914

\begin{abstract}
Recognition of pathogen-associated molecular patterns by Toll-like receptors (TLRs) activates innate and adaptive immune responses. Among the 11 members of the human TLR family, TLR-5 is known to play an important role in the defense against bacterial invasion by binding to flagellin, a conserved component of bacteria. Previous studies have demonstrated that the activation of TLR-5 induces the expression of interleukin (IL)-10, IL-12 and interferon- $\beta$. However, the aim of the present study was to analyze the expression of a wider range of immune-related molecules upon stimulation with a TLR-5 agonist. Following isolation from healthy volunteers, peripheral blood mononuclear cells (PBMCs) were stimulated with flagellin, a TLR-5 agonist. At $4 \mathrm{~h}$ after stimulation, quantitative polymerase chain reaction (PCR) and an antibody chip array were conducted to determine the mRNA expression levels of immune molecules and the protein secretion of immune molecules in the supernatant, respectively. The PCR results revealed that activation of TLR-5 significantly influenced the expression of a number of important molecules. In addition, the antibody chip array demonstrated the induction (IL-8) and inhibition [monocyte chemoattractant protein (MCP)-1, MCP-3 and macrophage inflammatory protein-1 $\alpha$ ) of protein secretion following TLR-5 stimulation. Therefore, the present study demonstrated the importance of TLR-5 in regulating the biological function of PBMCs. In the future, research should focus on the roles of the candidate molecules in TLR-5-mediating functions.
\end{abstract}

Correspondence to: Dr Quansheng Li, Department of Biliary Surgery, West China Hospital, Sichuan University, 37 Guo Xue Street, Chengdu, Sichuan 610041, P.R. China

E-mail: liquansheng@medmail.com.cn

Key words: Toll-like receptor-5, flagellin, peripheral blood mononuclear cells, quantitative polymerase chain reaction, antibody chip, cytokines, chemokines, tumor-associated genes

\section{Introduction}

Toll-like receptors (TLRs) are pattern recognition receptors that have important functions within innate immunity. TLRs can trigger a number of cellular processes, including the translocation of transcription factors, cytokine expression and the activation of protein kinases, through the recognition of conserved pathogen-associated molecular patterns, which ultimately lead to the induction of inflammatory responses (1). In total, 11 human TLRs have been identified, with the functions of human TLR-1-9 confirmed. Among the TLR members, TLR-1, -2, -4, -5 and -6 are located on the cell surface and are designed for the engagement of extracellular pathogens, while TLR-3, - - , - 8 and -9 are located on the endosome and recognize intracellular pathogen-derived products (2). TLRs play a critical role in defending against invaded pathogens by recognizing pathogen components and chemical synthesis. In addition, TLRs can protect the host from self-immune responses through the recognition of endogenous ligands released from damaged tissues (3).

TLR-5 has been demonstrated to play an important role in the defense against bacterial infection by binding to flagellin, the major protein found in bacterial flagella, which are critical for bacterial survival (4). TLR-5 is expressed on monocytes, dendritic cells, T lymphocytes, natural killer cells and epithelial cells, and provides a useful tool for the host to fight against the infection of flagellated bacteria. The activation of TLR-5 induces the secretion of a number of immune-associated molecules, including interleukin (IL)-2, IL-10 and IL-12, as well as type I interferons (IFNs) (5). However, a comprehensive list of the cytokines and chemokines induced following stimulation with a TLR-5 agonist has yet to be established.

In the present study, peripheral blood mononuclear cells (PBMCs) were isolated from healthy volunteers and stimulated with the TLR-5 agonist, flagellin. Subsequently, quantitative polymerase chain reaction (PCR) was performed to detect the expression levels of cytokines and chemokines in the PBMCs stimulated by flagellin. In addition, an antibody chip array was used to analyze the secretion of immune molecules in the supernatant of the flagellin-stimulated PBMCs. 


\section{Materials and methods}

Isolation of human PBMCs and stimulation with flagellin. In total, 5-ml blood samples were collected from healthy individuals (2 males, aged 25 and 27 years), and PBMCs were isolated using lymphocyte separation medium (MP Biomedicals, LLC, Santa Ana, CA, USA). The PBMCs were subsequently washed twice with phosphate-buffered saline. In total, $2 \times 10^{6}$ PBMCs were maintained in $10 \% 1640$ culture medium (Invitrogen, Carlsbad, CA, USA) and stimulated with $100 \mathrm{ng} / \mathrm{ml}$ flagellin (IMG2205; Novus Biologicals, LLC, Littleton, CO, USA), the TLR-5 agonist. The present study was approved by the Ethics Committee of the West China Hospital of Sichuan University (Chengdu, China). Written informed consent was obtained from all the individuals prior to their participation.

RNA extraction, reverse transcription and quantitative PCR. At $4 \mathrm{~h}$ after stimulation, the flagellin-treated and untreated control PBMCs were washed three times to remove any remaining flagellin. The total RNA was extracted using a RNeasy mini kit (Qiagen, Dusseldorf, Germany), and a ReverTra Ace reverse transcription kit (FSQ-101; Toyobo Co., Ltd., Kagoshima, Japan) was used to synthesize cDNA following the removal of genomic DNA by DNaseI (RT411; Tiangen Biotech Co., Ltd., Beijing, China). The conditions for the reverse transcription reaction were as follows: $65^{\circ} \mathrm{C}$ for $5 \mathrm{~min}$, followed by $37^{\circ} \mathrm{C}$ for $15 \mathrm{~min}$ and $98^{\circ} \mathrm{C}$ for $5 \mathrm{~min}$.

Quantitative PCR was performed using SYBR Green RealMasterMix (FP202; Tiangen Biotech Co., Ltd.). The quantitative PCR assay was performed in an iCycler iQTM Optical Module (Beckman Coulter, Inc., Fullerton, CA, USA) under the following conditions: Initial denaturation at $95^{\circ} \mathrm{C}$ for $30 \mathrm{sec}$, followed by 40 cycles of denaturation at $95^{\circ} \mathrm{C}$ for $30 \mathrm{sec}$, annealing at $58^{\circ} \mathrm{C}$ for $30 \mathrm{sec}$ and elongation at $72^{\circ} \mathrm{C}$ for $30 \mathrm{sec}$. A melting curve was constructed between temperatures of $55-95^{\circ} \mathrm{C}$, in $0.5^{\circ} \mathrm{C}$ increments and 10 -sec intervals. The primers used for quantitative PCR are shown in Table I, and glyceraldehyde 3-phosphate dehydrogenase was used as an internal control. Untreated PMBCs were used as a negative control, and all experiments were performed in triplicate.

Detection of secretory molecules using an antibody chip array. At $4 \mathrm{~h}$ after stimulation with flagellin, the cell supernatants were collected from the treated and untreated PBMCs. A RayBio ${ }^{\circledR}$ Human Antibody Array (C series; RayBiotech, Inc., Norcross, GA, USA) was applied for the screening of the secretory proteins, according to the manufacturer's instructions.

Statistical analysis. Data obtained from the quantitative PCR assay were analyzed using Bio-Rad iQ5 software (Bio-Rad Laboratories, Inc., Hercules, CA, USA). The results are expressed as the mean \pm standard error of the mean, and were statistically analyzed by a $\chi^{2}$ test using SPSS 17.0 software (SPSS, Inc., Chicago, IL, USA). $\mathrm{P}<0.05$ and $\mathrm{P}<0.001$ were considered to indicate a statistically significant difference. The figures were constructed using GraphPad Prism 5 software (GraphPad Software, Inc., La Jolla, CA, USA).

\section{Results}

Cytokine expression is induced by the activation of TLR-5. Expression levels of cytokines were analyzed using quantitative PCR, in order to determine the candidate molecules that were important in the TLR-5-mediated biological functions of PBMCs.

In the detection of cytokine expression, several important molecules were found to be highly expressed in the flagellin-stimulated PBMCs, which were IL-12 ( $\mathrm{P}<0.001)$, tumor necrosis factor- $\alpha(\mathrm{P}<0.001)$ and transforming growth factor (TGF)- $\beta$, as well as IL-2 and IFN- $\beta$ (P<0.05; Fig. 1$)$. By contrast, the expression levels of IL-1 $\beta(\mathrm{P}<0.001)$ and IFN- $\gamma$-induced protein (IP)-10 $(\mathrm{P}<0.05)$ were inhibited upon TLR-5 activation, while the expression levels of IL-6 and IL-15 were found to be unchanged. Notably, the expression levels of IL-8 $(\mathrm{P}<0.05)$, IFN- $\gamma(\mathrm{P}<0.001)$ and nuclear factor- $\kappa \mathrm{B}(\mathrm{P}<0.001)$ were markedly inhibited following stimulation with the TLR-5 ligand (Fig. 1). These results were in contrast to a former study, which demonstrated that increased expression levels of IL- 8 and IFN- $\gamma$ upon pathogen stimulation were important in the immune response against foreign invaders.

Variation in the expression levels of chemokines stimulated by flagellin. In the study, the expression levels of a number of important chemokines from the chemokine ( $\mathrm{C}-\mathrm{C}$ motif) ligand (CCL) and chemokine (C-X-C) ligand (CXCL) families were detected. The results demonstrated that flagellin stimulation significantly increased the expression levels of CCL19 $(\mathrm{P}<0.001)$, CCL20 $(\mathrm{P}<0.001)$, CCL26 $(\mathrm{P}<0.001)$, CCL28 $(\mathrm{P}<0.001)$, CXCL6 $(\mathrm{P}<0.001)$ and CXCL12 $(\mathrm{P}<0.001)$ when compared with the untreated cells (Fig. 2). However, inhibited expression was observed for CCL5 $(\mathrm{P}<0.05)$, CCL8 $(\mathrm{P}<0.001)$ and CCL17 $(\mathrm{P}<0.001)$, and the expression levels of CCL22 and CXCL2 were shown to remain at the same level as the control cells (Fig. 2).

Detection of tumor-associated gene expression upon TLR-5 activation. A number of molecules are known to play an important role in tumor initiation. The molecules selected for the study included the antitumor genes, P53 and phosphatase and tensin homolog (PTEN), the oncogenes, c-Myc and proliferating cell nuclear antigen (PCNA), as well as a number of important kinase signaling molecules, including $\beta$-catenin (CTNNB), c-Jun N-terminal kinase (JNK), mitogen-activated protein kinase (MAPK), mitogen-activated protein kinase kinase kinase 1 and phosphorylated signal transducer and activator of transcription (pSTAT3). The results indicated that the expression levels of P53 $(\mathrm{P}<0.001)$ and PTEN $(\mathrm{P}<0.001)$ were highly induced by flagellin, while c-Myc expression was inhibited $(\mathrm{P}<0.05$; Fig. 3). However, the expression levels of PCNA and vascular endothelial growth factor were not significantly influenced by TLR-5 activation. With regard to the kinase signaling molecules, induced expression was observed for MAPK and pSTAT3, while CTNNB and JNK expression levels were inhibited (Fig. 3).

Antibody chip array of the secretory molecules in the supernatant. Supernatants of the TLR-5-stimulated and 
Table I. List of oligonucleotides used for quantitative polymerase chain reaction detection.

\begin{tabular}{|c|c|c|c|}
\hline Gene & Forward primer & Reverse primer & GenBank number \\
\hline CCL5 & GACACCACACCCTGCTGCT & TACTCCTTGATGTGGGCACG & NM_002985 \\
\hline CCL8 & GTTTCTGCAGCGCTTCTGTG & TGGCTGAGCAAGTCCCTGA & Y10802 \\
\hline CCL17 & CCATCGTTTTTGTAACTGTGCAG & TGCATTCTTCACTCTCTTGTTGTTG & NM_002987 \\
\hline CCL19 & GGCACCAATGATGCTGAAGA & GAAGTTCCTCACGATGTACCCAG & NM_006274 \\
\hline CCL20 & TCCTGGCTGCTTTGATGTCA & TCAAAGTTGCTTGCTGCTTCTG & NM_004591 \\
\hline CCL22 & TGCGCGTGGTGAAACACT & GGTTAGCAACACCACGCCA & NM_002990 \\
\hline CCL26 & CCAAGACCTGCTGCTTCCAA & GAATTCATAGCTTCGCACCCA & NM_006072 \\
\hline CCL28 & CTCGCCATCGTGGCCTT & GCAATGGGAAGTATGGCTTCTG & AF220210 \\
\hline c-Myc & CAAGACTCCAGCGCCTTCTC & GTTGAGTAACGAGCTGACCCC & AM393287 \\
\hline CTNNB & CATCGTGAGGGCTTACTGGC & GAGCAAGGCAACCATTTTCTG & XM_006712984 \\
\hline CXCL2 & AGGTGAAGTCCCCCGGAC & GCCCATTCTTGAGTGTGGCT & NM_002089 \\
\hline CXCL6 & GCTGAGAGTAAACCCCAAAACG & GGAGCACTGCGGGCC & NM_002993 \\
\hline CXCL12 & GCCCGTCAGCCTGAGCTA & GACGTTGGCTCTGGCAACAT & NM_000609 \\
\hline GAPDH & GAAGGTGAAGGTCGGAGTC & GAAGATGGTGATGGGATTTC & $\mathrm{J} 04038$ \\
\hline IFN- $\beta$ & CAGCAATTTTCAGTGTCAGAAGCT & TCATCCTGTCCTTGAGGCAGT & M28622 \\
\hline IFN- $\gamma$ & CCAACGCAAAGCAATACATGA & CGCTTCCCTGTTTTAGCTGC & J00219 \\
\hline IL-1 $\beta$ & ACGAATCTCCGACCACCACT & CCATGGCCACAACAACTGAC & M15330 \\
\hline IL-2 & CAAGAATCCCAAACTCACCAGG & GACACTGAAGATGTTTCAGTTCTGT & J00264 \\
\hline IL-6 & GACCCAACCACAAATGCCA & GTCATGTCCTGCAGCCACTG & M14584 \\
\hline IL-8 & CTGGCCGTGGCTCTCTTG & CCTTGGCAAAACTGCACCTT & NM_000584 \\
\hline IL-12 & CGGTCATCTGCCGCAAA & CAAGATGAGCTATAGTAGCGGTCCT & M65272 \\
\hline IL-15 & GACCCCACCAAAGCTGGAC & TCACAGTGCTGCTGTCTGCTG & M90391 \\
\hline IP-10 & TGAAATTATTCCTGCAAGCCAA & CAGACATCTCTTCTCACССТTCTTT & NM_001565 \\
\hline JNK & GCTAATTCTGTACCAATGTC & GAAGAGTGCACGTCAGGAAC & NM_139049 \\
\hline MAPK & CCTGCTCGGTGCACGATGCTG & CTCTGTCTCTTCACGTGGCGG & NM_003954 \\
\hline MEKK1 & CTTTTAAGTCAGAAGTTGCTG & СТTCTCCATTTTCAACCTGC & AF042838 \\
\hline$N F-\kappa B$ & AGAGTGCTGGAGTTCAGGATA & AAGGTGGATGATTGCTAAGTGT & AJ271718 \\
\hline P53 & CTTGCATTCTGGGACAGCCAAG & CACGCAAATTTCCTTCCACTCGG & DQ892492 \\
\hline PTEN & ACCATAACCCACCACAGC & CAGTTCGTCCCTTTCCAG & NM_058074 \\
\hline pSTAT3 & CCTACAAAGGGGACCCCATTGTAC & CAGGGAATTTGACCAGCAACC & NM_213662 \\
\hline TGF- $\beta$ & TATCGACATGGAGCTGGTGAAG & CAGCTTGGACAGGATCTGGC & $\mathrm{X} 02812$ \\
\hline $\mathrm{TNF}-\alpha$ & GGTGCTTGTTCCTCAGCCTC & CAGGCAGAAGAGCGTGGTG & M10988 \\
\hline VEGF & GACTTGAGTTGGGAGGGGAA & GAGGCTCAGCGCCAGGGCTGGG & AF024710 \\
\hline
\end{tabular}

CCL, chemokine (C-C motif) ligand; CTNNB, $\beta$-catenin; CXCL, chemokine (C-X-C motif) ligand; GAPDH, glyceraldehyde 3-phosphate dehydrogenase; IFN, interferon; IL, interleukin; IP, INF- $\gamma$-induced protein; JNK, c-Jun N-terminal kinase; MAPK, mitogen-activated protein kinase; MEKK, mitogen-activated protein kinase kinase kinase; NF, nuclear factor; PTEN, phosphatase and tensin homolog; pSTAT, phosphorylated signal transducer and activator of transcription; TGF, transforming growth factor; TNF, tumor necrosis factor; VEGF, vascular endothelial growth factor.

unstimulated PBMCs were collected, and the detection of the secreted molecules was performed using a RayBio ${ }^{\circledR}$ antibody chip array. In total, 20 important cytokines and chemokines were included in the array. The results indicated that only four molecules produced a positive response. Among these molecules, the secretion of monocyte chemoattractant protein $(\mathrm{MCP})-1(\mathrm{P}<0.05), \mathrm{MCP}-3(\mathrm{P}<0.05)$ and macrophage inflammatory protein-1 $\alpha$ were significantly inhibited (Fig. 4). However, the secretion of IL-8 was identified in the supernatant, which contradicted with the results from the quantitative PCR that indicated that IL-8 expression was inhibited by flagellin stimulation. An explanation for this phenomenon is that changes in gene expression are not always consistent with those in protein secretion.

\section{Discussion}

TLRs comprise a family of transmembrane proteins that differentially recognize pathogens and initiate inflammatory signaling pathways (6). An important function of TLRs is their ability to bind to microorganisms by recognizing conserved structures, which are known as pathogen-associated molecular patterns (7). All members of the TLR family are able to trigger a specific immune response by activating MYD88-mediated 


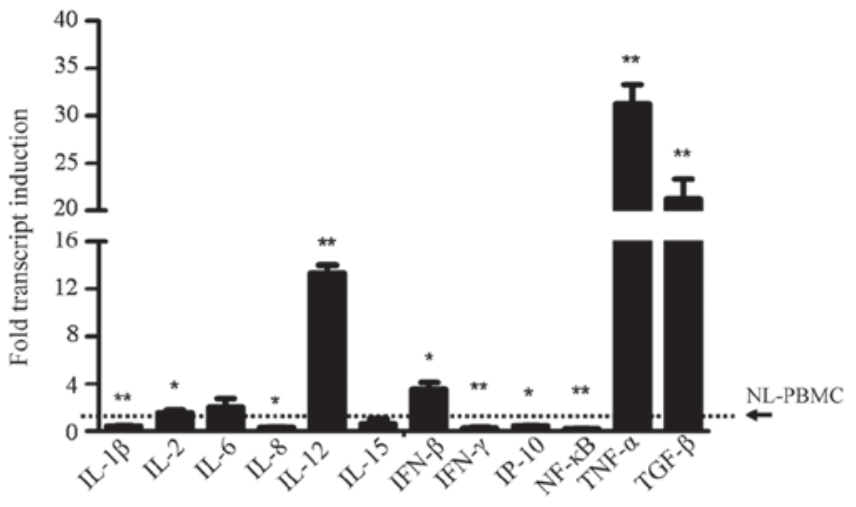

Figure 1. Detection of cytokine expression using quantitative polymerase chain reaction. ${ }^{* *} \mathrm{P}<0.001$ and ${ }^{*} \mathrm{P}<0.05$, vs. control group. In quantitative polymerase chain reaction analysis, the level of NL-PBMC was set as the control group and the expression level was set as 1. IL, interleukin; IFN, interferon; IP, IFN- $\gamma$-induced protein; NF, nuclear factor; TNF, tumor necrosis factor; TGF, transforming growth factor; NL-PMBC, normal peripheral blood mononuclear cells.

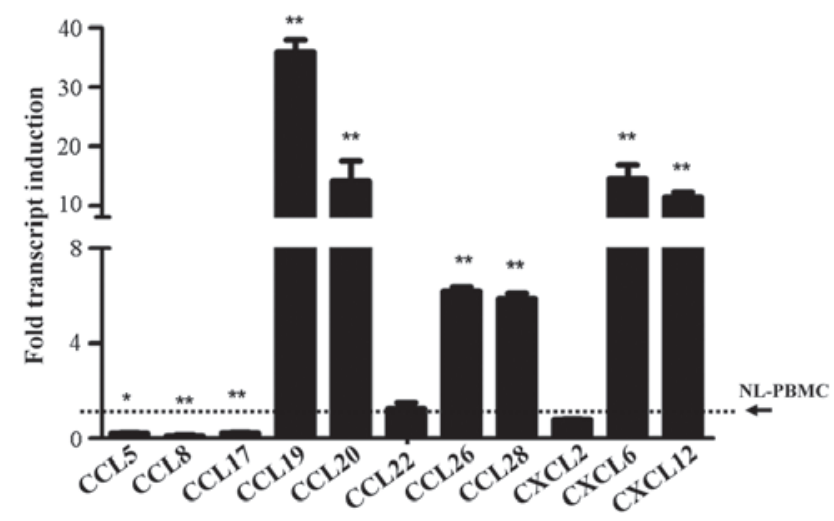

Figure 2. A quantitative polymerase chain reaction assay was used to analyze the expression levels of chemokines. ${ }^{* *} \mathrm{P}<0.001$ and ${ }^{*} \mathrm{P}<0.05$, vs. control group. In quantitative polymerase chain reaction analysis, the level of NL-PBMC was set as the control group and the expression level was set as 1. CCL, chemokine (C-C motif) ligand; CXCL, chemokine (C-X-C motif) ligand; NL-PMBC, normal peripheral blood mononuclear cells.

pathways, with the exception of TLR-3, which subsequently induces a number of immune molecules that are important in disease treatment (8). TLR-5 has been shown to function in the defense against invading microbes via the recognition of flagellin on bacteria (9). A previous study demonstrated that activation of TLR-5 increased the expression levels of IL-10 and IL-12, while inhibiting IFN- $\gamma$ expression (10).

In the present study, PBMCs collected from healthy volunteers were treated with the TLR-5 ligand, flagellin. Subsequently, the variation in the expression levels of immune molecules was detected to determine which molecules were important for the biological functions of PMBCs, including tumor initiation, the inflammatory response and cell proliferation. The results demonstrated that flagellin stimulation induced the expression of IL-12, IFN- $\beta$ and TGF- $\beta$, which were consistent with the results of a previous study (11). The major difference between the present and previous studies was that the current study covered a wider range of molecules that are known to be important in mediating the biological functions of PBMCs, including

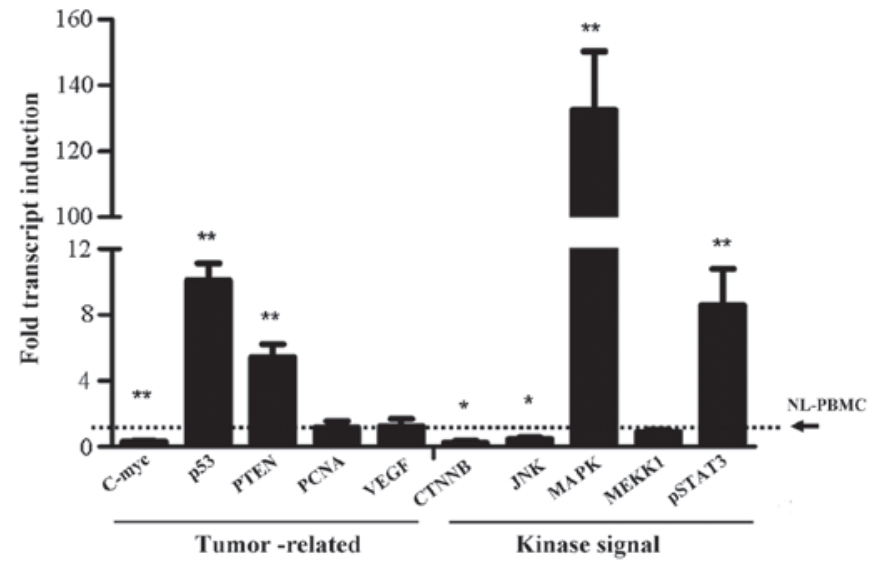

Figure 3. Variation in the expression levels of tumor-associated genes and kinase signaling molecules, as assessed by quantitative polymerase chain reaction. ${ }^{* *} \mathrm{P}<0.001$ and ${ }^{*} \mathrm{P}<0.05$, vs. control group. In quantitative polymerase chain reaction analysis, the level of NL-PBMC was set as the control group and the expression level was set as 1. PTEN, phosphatase and tensin homolog; PCNA, proliferating cell nuclear antigen; VEGF, vascular endothelial growth factor; CTNNB, $\beta$-catenin; JNK, c-Jun N-terminal kinase; MAPK, mitogen-activated protein kinase; MEKK, mitogen-activated protein kinase kinase kinase; pSTAT, phosphorylated signal transducer and activator of transcription.

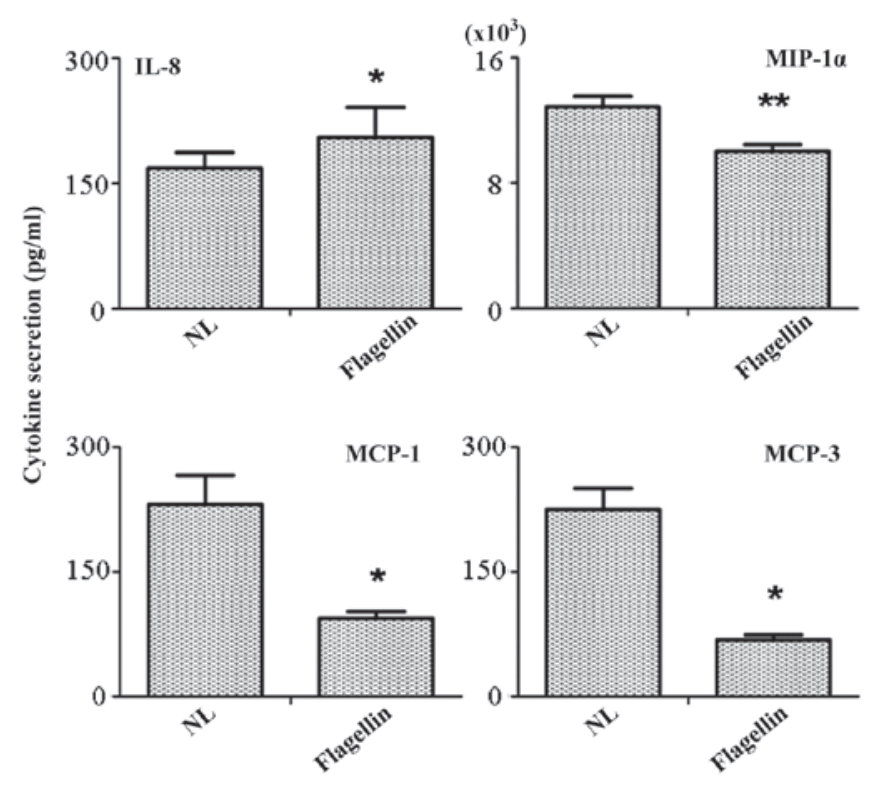

Figure 4. An antibody chip array was used to assess the secretion of cytokines and chemokines in the supernatant. ${ }^{* *} \mathrm{P}<0.001$ and ${ }^{*} \mathrm{P}<0.05$, vs. control group. NL, normal peripheral blood mononuclear cells; IL, interleukin; MIP, macrophage inflammatory protein; MCP, monocyte chemoattractant protein.

molecules that influence the proinflammatory status, genes that mediate the antitumor response and kinase signaling molecules. The results of the present study confirmed that activation of the TLR-5 pathway inhibited the expression of IL-1 $\beta$, IL- 8 and IP-10, as well as the chemokines, CCL5, CCL8 and CCL17, indicating the unique role of TLR-5 in regulating the functions of PBMCs. In addition, the present study revealed increased expression levels of the antitumor genes, P53 and PTEN, and the kinase signaling molecules, MAPK and pSTAT3, in the TLR-5 stimulated cells, indicating the potential role of TLR-5 in the antitumor response. 
Although the present study has demonstrated that a number of interesting candidate molecules are involved in the mechanism of TLR-5, a limitation of the study was the short treatment time (4 h) of flagellin, which was selected as this is the common stimulation time in the presence of a TLR agonist (12). In addition, certain important molecules exhibited no response upon ligand stimulation, particularly in the antibody chip array, with only four molecules showing a positive response in an array of $>20$ molecules. Therefore, further study should aim to investigate the roles of the candidate molecules, identified in the present study, in mediating the biological functions of PBMCs.

\section{References}

1. Akira S, Uematsu S and Takeuchi O: Pathogen recognition and innate immunity. Cell 124: 783-801, 2006.

2. Takeda K, Kaisho T and Akira S: Toll-like receptors. Annu Rev Immunol 21: 335-376, 2003.

3. Ozinsky A, Underhill DM, Fontenot JD, et al: The repertoire for pattern recognition of pathogens by the innate immune system is defined by cooperation between Toll-like receptors. Proc Natl Acad Sci USA 97: 13766-13771, 2000.
4. Iwasaki A and Medzhitov R: Regulation of adaptive immunity by the innate immune system. Science 327: 291-295, 2010.

5. Merio A, Calcaterra C, Mènard S and Balsari A: Cross-talk between Toll-like receptors 5 and 9 on activation of human immune responses. J Leukoc Biol 82: 509-518, 2007.

6. Akira S and Takeda K: Toll-like receptor signalling. Nat Rev Immunol 4: 499-511, 2004.

7. Beutler B: Inferences, questions and possibilities in Toll-like receptor signaling. Nature 430: 257-263, 2004.

8. Balander JM and Medzhitov R: Regulation of phagosome maturation by signals from Toll-like receptors. Science 304: 1014-1018, 2004.

9. Hawn TR, Verbon A, Letinga KD, et al: A common dominant TLR5 stop codon polymorphism abolishes flagellin signaling and is associated with susceptibility to legionnaires' disease. J Exp Med 198: 1563-1572, 2003.

10. Hayashi F, Smith KD, Ozinsky A, et al: The innate immune response to bacterial flagellin is mediated by Toll-like receptor 5. Nature 410: 1099-1103, 2001.

11. Andersen-Nissen E, Smith KD, Strobe KL, et al: Evasion of Toll-like receptor 5 by flagellated bacteria. Proc Natl Acad Sci USA 102: 9247-9252, 2005.

12. Lin Y, Zhang L, Cai AX, Lee M, Zhang W, Neuberg D, Canning CM, Soiffer RJ, Alyea EP, Ritz J, Hacohen N, Means TK and Wu CJ. Effective posttransplant antitumor immunity is associated with TLR-stimulating nucleic acid-immunoglobulin complexes in humans. J Clin Invest 121: 1574-1584, 2011. 